\title{
DSR and OLSR routing protocol based performance evaluation and integration on MIP with MANET
}

\author{
Sirajum Munira, Sadia Humaira, Samara Fahin, Ashraf Siddik, A.K.M.Fazlul Haque \\ Department of ETE, Daffodil International University, Bangladesh
}

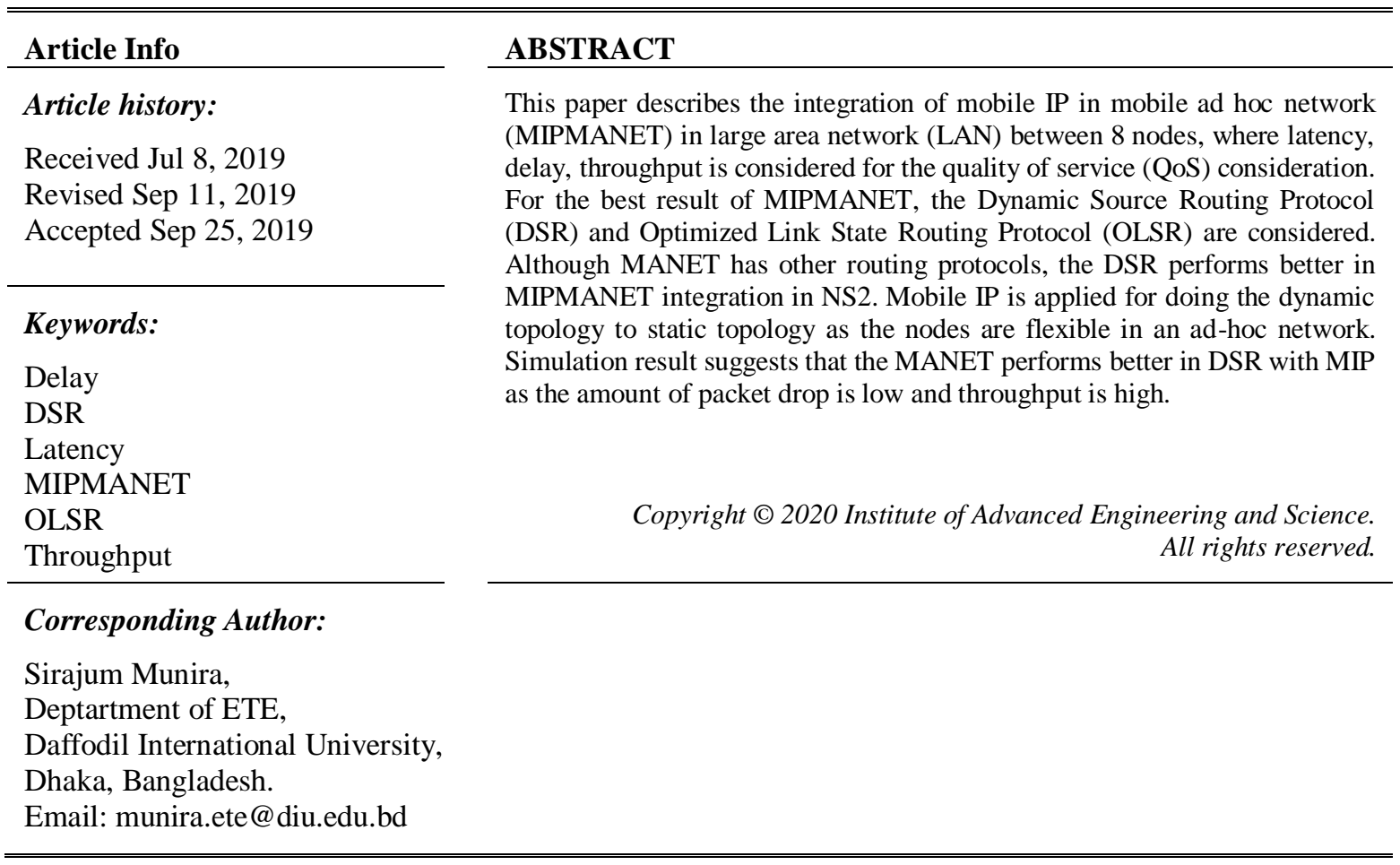

\section{INTRODUCTION}

Presently varieties of systems with varieties of targets are co-existing under the heterogeneous condition. It is necessary from the client perspective to have the persistent method of system availability and comprehensive forthright to every one of the clients. In the meantime, a new application requests more transmission capacity and lower costs. Therefore, a great deal of scheming has been produced among the analyst in recent years giving a ubiquitous correspondence between divergent systems under heterogeneous condition. Our point is to concentrate such endeavors of fusion utilizing the system layer conventional Mobile IP. The test bed has been established using NS-2 for reformation. NS experiences various incongruences and weaknesses, which limit its use and advancement of conventions for new situations in wired, remote, portable and identified with future heterogeneous systems. The point in this paper is to show a reasonable and summed up technique for executing versatile IP convention [1].

IEEE 802.11 standard depends on Wireless neighborhood (WLAN) and has turned out to be exceptionally prominent as the remote system requests increment. WLAN offers rapid (up to $54 \mathrm{Mbps}$ ) contrast with different remote principles with higher client mobility highlights with minimal effort. WLAN likewise reinforces a wide range of utilization like sight and sound and voice that utilize IEEE 802.11 conventions, for example, VOIP, video conferencing, live broadcast, video spilling mixed media gushing and so on. Quality of Services (QoS) is a stand out amongst the most imperative parameters to be considered while giving and supporting these mixed media and ongoing administrations to end-clients [2]. Handoff is the way toward changing the channel (recurrence, vacancy, spreading code, or the mix of them) related to the present association while a call is in advancement. It is regularly started either by intersection of a phone limit or by crumbling in nature of the flag in the present channel. 
Communication has moved from a transcendently wired setup towards a completely remote setup, or a merger. PC systems help in quicker and dependable interchanges over long separations. The Internet, a system of systems, has turned into an essential utility in our lives that empower us to impart far and wide [3]. Versatility as an element in correspondence has picked up the acknowledgment of end-clients. In this way, it isn't amazing that Mobile Ad hoc Networks (MANETs) have pulled in much consideration from specialists. A MANET is a self-sufficient, framework less, self-forming and self-fixing information system of cell phones that help multi-jump correspondence. MANET could be utilized to give Internet availability past the scope of settled or cell framework [1].

On the Internet (IP) condition, when a portable hub moves and connects itself to another system, it needs to acquire another IP address. This changing of IP address implies that all current IP associations with the versatile hub should be ended and afterward restored. This is fundamental as the IP steering systems depend on the topological data implanted in the IP delivery to convey the information to the right end-point. Portable IP (MIP) depicts a worldwide arrangement that conquers this issue using indirection given by a lot of system operators. It doesn't require any alterations to existing switches or end reporter hubs. With MIP, every portable hub is distinguished by a location from its home system, paying little respect to the point of connection. While a portable hub is far from its home system, it gets an IP address from the meeting system and registers it with a home operator inside its home system [4]. The home operator blocks any parcels bound to the portable hub, and burrows or expressly courses (source steering) them to the versatile hub's present area. Subsequently, starting this indirection requires an opportune location reconfiguration system and a home system enlistment process. The time is taken for a portable hub to arrange another system care-of location in the meeting system, and the time taken to enroll with the home specialist together establish the (generally) handoff dormancy [5].

Mobile IP is the present standard for supporting IP mobility of mobile hubs in the remote systems with the framework. Mobile IP empowers the portable hub to get to the Internet and changes its passageway without losing the association. The mobile hub ought to be in the inclusion scope of Mobile IP base station (passage) and has an immediate association with it [3]. MANET is a sort of remote system design that can be adaptably conveyed in any condition (e.g., gathering rooms, backwoods, front lines, and so on.) without the need of system framework or concentrated organization. MANET clients can get to the Internet and access an extensive variety of Internet administrations and applications [1]. Due to the restricted inclusion of MANETs, joining of MANETs with the settled foundation IP get to the network can build this inclusion. Joining of MANETs with the settled framework IP get to arrange dependent on IP portability conventions empowers MANET hubs development between various MANETs without losing the association. It can boost portability between various non-overlapping and covering MANETs with different doors [6].

In the present usage of wireless networks, when a node moves to start with one passageway then onto the next passageway, it restores the association each time with an alternate IP address. This expands the Latency of the system and furthermore gives an intruded-on administration. This sort of correspondence can be productively actualized utilizing Mobile IP. Mobile IP, which is an expansion to standard Internet Protocol proposed by the Internet Engineering Task Force (IETF) [7]. It keeps up a similar IP address notwithstanding when the host node moves from one system to the next. While enlisting with the home operator Mobile IP could be reached out to envelop every one of the advancements for consistent portability if the accompanying issues are settled. These are Security Issues, Triangulation Problems, Reliability Issues, and Latency Issues $[8,9]$.

In between two modes of wireless networks, ad hoc is the easiest way to design anywhere anytime for emergency situation. When devices or products are connected by internet of services or IoT there will be happened many problems like increasing latency or delay. So, latency is the concern for devices that can run effectively. As mobile ad hoc is dynamic topology that's why mobile IP is useful as it has static IP. So, when one mobile host pass away to another through foreign network there will be handoff. So, it is important to simulate how mobile IP works with the increasingly expanded mobile usage in mobile ad hoc network. The main focus of this paper is to design and analyze solutions for using mobile IP on Dynamic and Static routing protocol in mobile ad hoc network with reduced delay in MIPMANET integration. Besides, the quality of service $(\mathrm{QoS})$ has been measured for this network.

\section{LITERATURE REVIEW}

In the different research paper various work has been done about the MANET and mobile IP. A mobile ad hoc network is an infrastructures network, self-configuring where devices connected wirelessly. The correlation between the Mobile ad hoc routing protocol and mobile IP is important for communication in the large area network. The connectivity with the internet changing its access point without losing the connection is the main concern of mobile IP. Many solutions have been proposed to access internet with mobile IP in the MANET. Fekri M.Abdullah et al described a review of answers for incorporating MANETs with the 
internet, with the plan of filling in as a speedy reference to momentum inquire about proposition for internet availability for coordination is exhibited [10]. Kingsley K. Ofosu et al described the execution of mobile IP on MANET in network simulator 2 (NS2). Ad hoc On-demand Multiple Distance Vector (AOMDV) and Destination-sequenced Distance Vector (DSDV) routing protocols were used to measure throughput, End to End Delay(E2ED), packet delivery ratio (PDR) and normalized packet ratio (NPR) [1]. Nima Sarshar et al described the scaling of the quantity of hops in wireless ad hoc network (WANET) including the problem of network latency, packet loss, power and memory [11]. Fredrik Alrisson et al described about MIPMANET to provide internet access by using mobile IP with foreign agent care-of addresses and reverse tunneling and AODV routing protocol was used for routing within the ad hoc network [7, 12]. While there is a set of protocols to improve the quality of service for MANET, it has several limitations. I. Varshney et al. have shown different ways that can reduce the limitation of MANET [13-20].

\section{METHODOLOGY}

Network Simulator 2 is an open-source case-driven test system structured explicitly for research in PC correspondence systems. It has persistently increased gigantic enthusiasm from industry, the scholarly world, and government. Having been under consistent examination and improvement for a considerable length of time, in this simulator now contains modules for various system segments, for example, directing, transport layer convention, application, and so on [21,22].

In Figure 1 it is shown that the network topology of mobile ad hoc network consists of 10 nodes. The source is node $n 0$ and $n 1$ where the destination is $n 5$ and $n 8$. Where TCP and UDP protocol is used to send data. Figure 2 shows that when a packet is sent to the destination and it is not found in the outing table, then broadcast process will be started and discover the rout path for the destination.

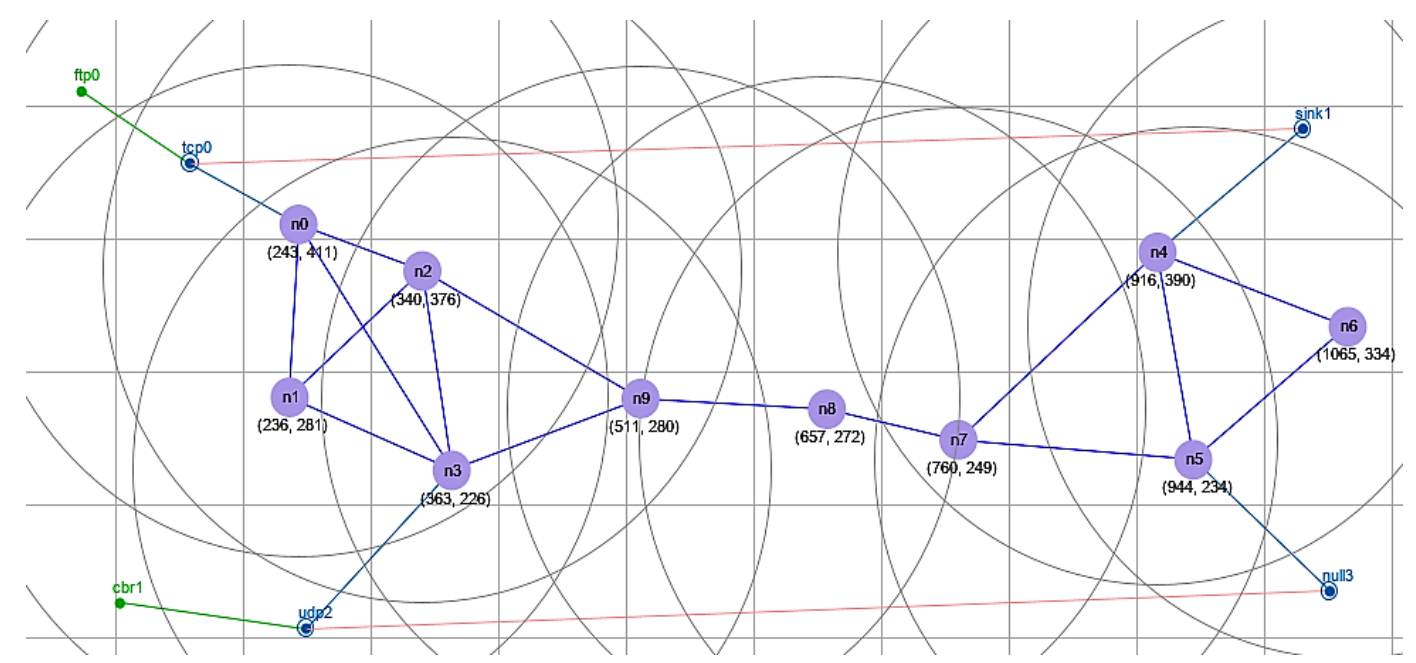

Figure 1. MIPMANET simulation topology

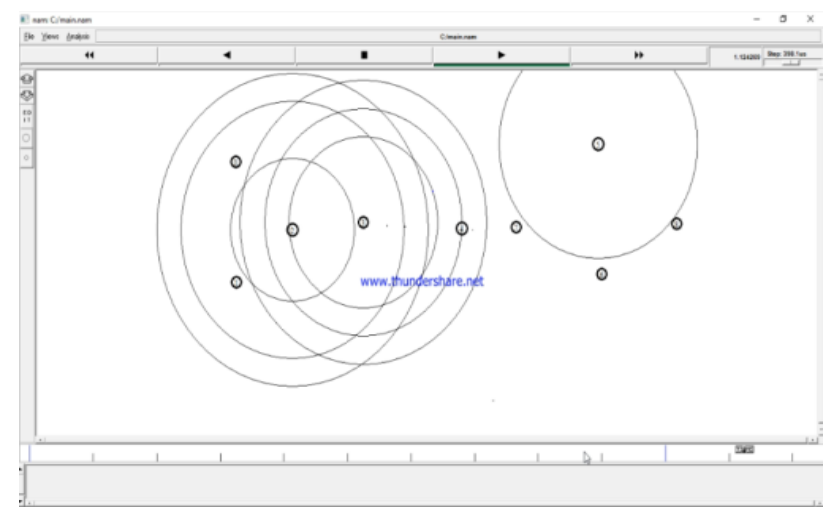

Figure 2. Route discovery

Indonesian J Elec Eng \& Comp Sci, Vol. 17, No. 3, March 2020 : 1306 - 1312 


\subsection{Performance Parameters}

This paper focuses on five aspects for the assessment of the study on the overall system performance. These aspects are:

a) Throughput is defined as; the ratio of the total data reaches a receiver from the sender. Throughput is expressed as bytes or bits per sec (byte/sec or bit/sec). A high throughput is absolute choice in every network. Throughput can be represented mathematically as in equation [23].

b) Throughput $=($ Number of Delivered Packet*Packet Size*8)/ Total Duration of Simulation

c) Delay is the average time it takes a data packet to reach the destination. This metric is calculated by subtracting transmission time from receiving time. This includes all possible delays caused by buffering during route discovery latency, queuing at the interface queue, retransmission delays at the MAC, propagation and transfer times.

d) Latency in network is measured as either one-way or round-trip delay time. One-way latency is measured as the time from the source sending a packet to the destination receiving it. And round-trip delay is the one-way time from source to destination plus the time from destination to the source [24].

e) Packet drop occurs when one or more packets of data travelling across a network fail to reach their destination [23].

\section{SIMULATION RESULTS}

The simulations have been performed utilizing Network Simulator 2 (Ns-2) especially mainstream in the specially appointed systems administration network. The source-goal sets have been spread haphazardly over the system. During the restoration, every hub begins its quest from an arbitrary spot to an irregularly chosen destination. When the destination has been reached, the hub takes a rest for a timeframe in second and another irregular destination is picked after that stop time. This procedure rehashes all through the regeneration, causing perpetual changes in the topology of the basic system $[25,26]$. Table 1 depicts the simulation parameters and specifications considered for the paper.

Table 1. Simulation Parameters and Specifications

\begin{tabular}{cc}
\hline Simulation Parameter & Value \\
\hline Channel Type & Wireless Channel \\
Radio-propagation model & Two Ray Ground Model \\
Network interface type & Wireless Physical \\
MAC type & $802 \_11 \mathrm{~b}$ \\
Interface Queue Type & Drop Tail Primary Queue \\
Antenna model & Omni Direction \\
Number of Mobile nodes & 10 \\
Ad Hoc Routing Protocol & DSR \& OLSR \\
Simulation Area & 500m x 400m \\
Simulation Time & $150 \mathrm{MS}$ \\
Traffic Type & TCP, UDP \\
Nodal speed & $3-10 \mathrm{~m} / \mathrm{s}$ \\
Packet size & 1000 Byte (Data Packets) \\
\hline
\end{tabular}

\subsection{Throughput}

One of the most crucial criteria for analyzing the performances of different routing protocols. Table 2 lists out the throughputs (bit/sec) and simulation times for different time scale for DSR and OLSR routing protocols.

Table 2. Data Table of Throughput

\begin{tabular}{|c|c|c|c|c|}
\hline \multirow[t]{2}{*}{ Time(s) } & \multicolumn{2}{|c|}{ DSR } & \multicolumn{2}{|c|}{ OLSR } \\
\hline & Throughput(bit/sec) & Simulation Time & Throughput(bit/sec) & Simulation Time \\
\hline 10 & 52800 & 0.09152117 & 15200 & 0.070694 \\
\hline 20 & 45600 & 0.03039 & 26000 & 0.059664 \\
\hline 30 & 27200 & 0.0188125 & 7600 & 0.029186 \\
\hline 40 & 39200 & 0.0259228 & 12800 & 0.022042 \\
\hline 50 & 28800 & 0.2278873 & 15000 & 0.01382 \\
\hline
\end{tabular}


Figure 3 shows the comparison of throughput between DSR and OLSR routing protocols in MIPMANET. It is clear from the graph that, the throughput of DSR routing protocol is quite higher than OLSR routing protocol. So, it can be observed the better performance of DSR over OLSR.

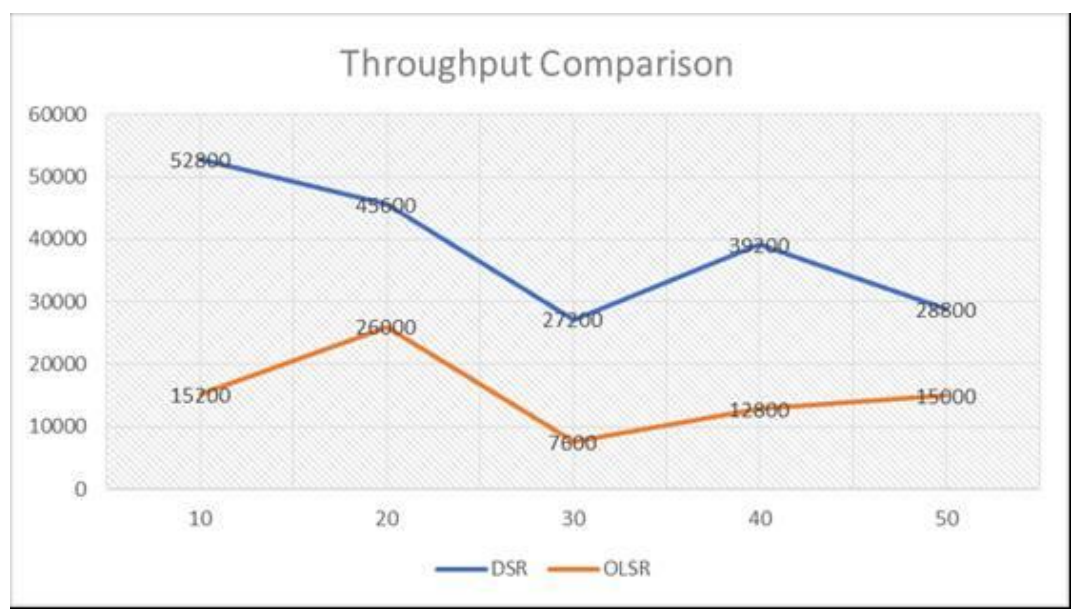

Figure 3. Comparison of throughput between OLSR and DSR

\subsection{End to End Delay}

End to End delay is the time taken for transmitting a packet from source to destination. In this paper, the simulation time has been measured between each nodes individually. Table 3 lists out the simulation time between each individual nodes for DSR and OLSR routing protocol.

The graph in the Figure 4 shows that the end to end delay between the same node is higher in the OLSR routing protocol where DSR routing protocol has lower end to end delay.

Table 3. Data Table of End to End Delay

\begin{tabular}{ccc}
\hline Node & Simulation Time (DSR) & Simulation Time (OLSR) \\
\hline 1,2 & 0.0007205 & 0.0008485 \\
5,7 & 0.0003527 & 0.0003047 \\
2,3 & 0.0005325 & 0.0003525 \\
4,7 & 0.0001200 & 0.0007844 \\
1,3 & 0.0010490 & 0.0007493 \\
3,4 & 0.0003527 & 0.0003526 \\
4,3 & 0.0003047 & 0.0003047 \\
1,6 & 0.0003142 & 0.0002249 \\
6,8 & 0.0003526 & 0.0003526 \\
1,8 & 0.0002281 & 0.0004111 \\
\hline
\end{tabular}

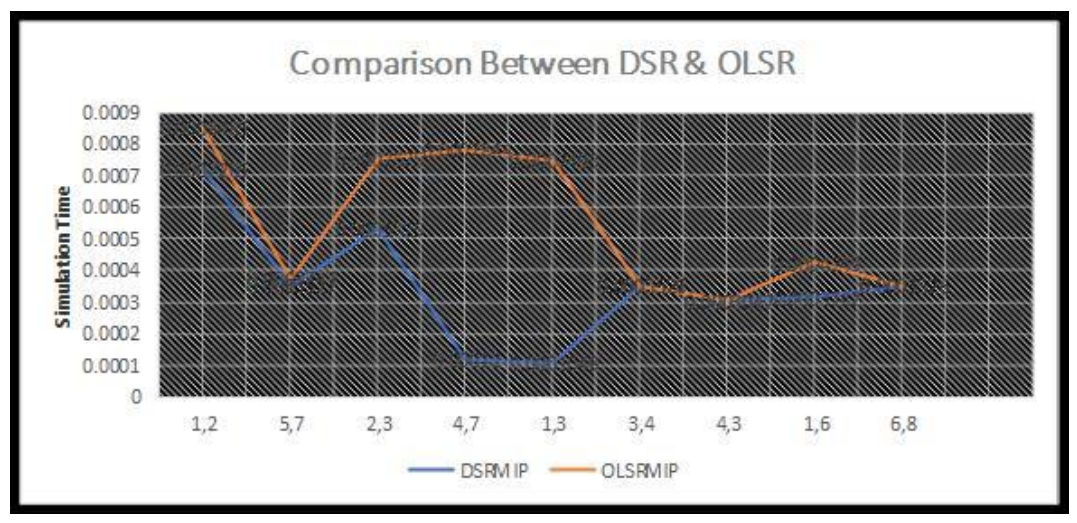

Figure 4. Comparison of DSRMIP and OLSRMIP 


\subsection{Latency}

Latency is measured by round trip time by sending a packet from source to the destination and returned to the source. One-way latency is measured as the time from the source sending a packet to the destination receiving it. And round-trip delay is the one-way time from source to destination plus the time from destination to the source [24]. Table 4 provides the list of latency for each individual pair of nodes for both DSR and OLSR routing protocols.

Table 4. Data Table of DSR

\begin{tabular}{lll}
\hline Node & Simulation Time ( DSR) & Simulation Time (OLSR) \\
\hline 6,8 & 0.0006572 & 0.0006564 \\
5,8 & 0.0006575 & 0.0006568 \\
7,6 & 0.0006573 & 0.0006565 \\
4,7 & 0.0006567 & 0.0006563 \\
7,5 & 0.0006575 & 0.0006562 \\
2,3 & 0.000657 & 0.0006561 \\
0,2 & 0.0006571 & 0.0006560 \\
1,2 & 0.000657 & 0.0006560 \\
\hline
\end{tabular}

In Figure 5 the comparison of latency for DSR and OLSR is shown. It is clearly shown that latency of OLSR is higher than DSR. By the meaning of this, DSR outperforms better than OLSR routing protocol in MIPMANET.

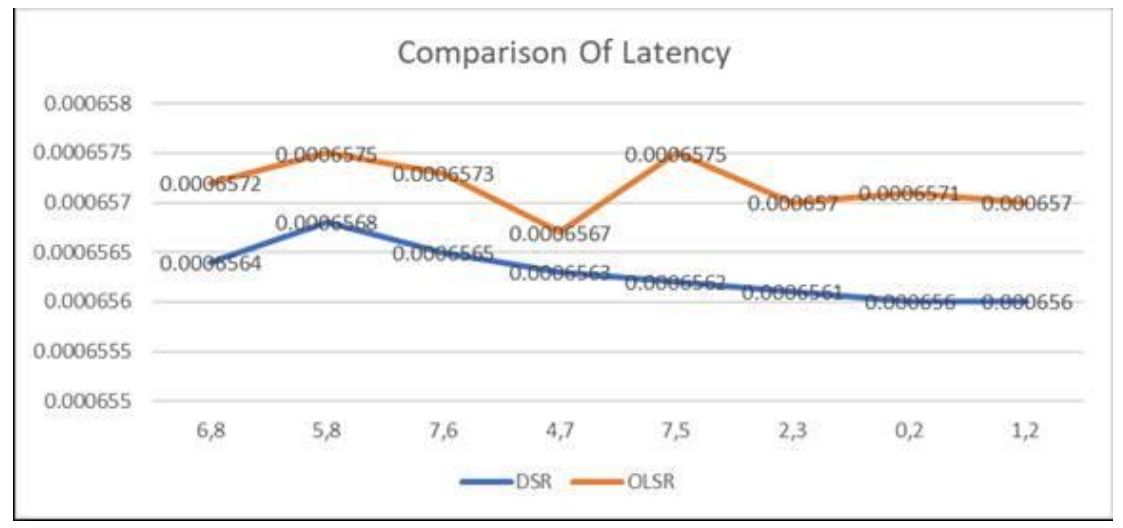

Figure 5. Comparison between DSR and OLSR

\subsection{Analysis}

According to the performance of the designed system of MIPMANET, the throughput of both DSR and OLSR routing protocol can be found. The performance of the routing protocols are measured by the quantity of the packets sent by the protocols. DSR routing protocol has higher number of delivered packets per second. In case of end to end delay, DSR has less end to end delay. Besides, according to the latency, DSR outperforms most than the other one. So, it has been finally decided by analyzing the performances, DSR routing protocol outperforms most than OLSR routing protocol in MIPMANET.

\section{CONCLUSION}

A performance assessment of Mobile IP on MANET using Dynamic and Static Routing convention has been used for directing packets in the MANET system. The performance of Mobile IP utilizing the DSR protocol on mobile ad hoc network outperforms better than OLSR. It has been seen that a few recommendations have attempted to help reduced scale mobility by coordinating Mobile IP with MANETs, and just a single of them has applied to use the advantages of the reduced scale portability protocol. Simulation results have been analyzed from the execution of Mobile IP on MANET with respect to normal end-to-end delay, throughput, latency, and standardized packet quantity. The overall performance of DSR has better acceptance for all measurement than OLSR. 


\section{REFERENCES}

[1] K. K. Ofosu, J. D. Abdulai, and F. A. Katsriku, "Mobile IP on Mobile Ad Hoc Networks: An Implementation and Performance Evaluation Using NS2," Advanced Computing: An International Journal (ACIJ), vol. 9, no. 2, 2018.

[2] M. L. Tetarwal, A. Kuntal, and P. Karmakar, "A Review on Handoff latency Reducing Techniques in IEEE 802," 11 WLAN, In IJCA Proceedings on National Seminar on Recent Advances in Wireless Networks and Communications (No. 2, pp. 22-28). Foundation of Computer Science (FCS), April 2014.

[3] Falade, Olumuyiwa T. \&Botsio, Marcellus "Mobile IP handover for WLAN" Technical report, IDE1015, March 2010.

[4] Babak Ayani "Smooth Handoff in Mobile IP," 2002-05-14.

[5] A. Diab, and A. Mitschele-Thiel. "Minimizing Mobile IP Handoff Latency," 2nd International Working Conference on Performance modelling and Evaluation of Heterogeneous Networks (HET-NETs' 04), Ilkley, West Yorkshire, UK, 2004

[6] S. Aust, N. A. Fikouras, M. Sessinghaus, C. Görg, and C. Pampu, "Hierarchical Mobile IP NS-2 Extensions for Mobile Ad Hoc Networks," Proceedings of Wireless Networks and Emerging Technologies, 2004.

[7] F. Alriksson, U. Jönsson, and K. Purser, "Mobile IP for mobile ad hoc networks." U.S. Patent 6,977,938, issued December 20, 2005.

[8] http://www.computerscijournal.org/vol10no2/major-challenges-of-mobile-adhoc-networks.

[9] Laureano, R. M., Fernandes, A. L., Hassamo, S., \& Alturas, B. (2018). Facebook satisfaction and its impacts on fundraising: a case study at a Portuguese non-profit organization. Journal of Information Systems Engineering and Management, (1).

[10] F. A. Bodhe, "Ip Mobility Protocols and Mobile Adhoc Networks," ISTQUARTER, vol. 9, no. 1, IEEE communication surveys, 2007.

[11] N. Sarshar, B. A. Rezaei, and V. P. Roychowdhury, "Low Latency Wireless Ad-Hoc Networking: Power and Bandwidth Challenges and a Hierarchical Solution," arXiv preprint cs/0604021, 2006.

[12] J., Yakubu H., Aboiyar T., and Zirra P. B.. "An improved RSA image encryption algorithm using 1-D logistic map." International Journal of Communication and Computer Technologies 6.1 (2018), 1-6.

[13] I. Varshney, G. Ananth Kumar, "Study of Mobile Ad-hoc Network's Challenges and Characteristics," Int J Sci Res Sci Technol., Vol.6, Issue 4, July-August 2019.

[14] S..O.A., S. R., A. Q.R., N. Faruk, O. L. A., A. T.C., O. A.A, "Comparative Study of Routing Protocol Performance in Mobile Ad hoc Network," International Journal of Information Processing and Communication (IJIPC), Vol. 7, No. 1, May 2019.

[15] K. Singh and R. Gupta, "Comparative Assessment and Performance Analysis of Numerous Mobile Ad-Hoc Network Routing Protocols," Journal of Computational and Theoretical Nanoscience Vol. 16, 3906-3911, 2019.

[16] Y.Chalapathi Rao, P.Kishore, S.Rajendra Prasad, "Riverbed Modeler Simulation-Based Performance Analysis of Routing Protocols in Mobile Ad Hoc Networks," International Journal of Recent Technology and Engineering (IJRTE), ISSN: 2277-3878, Volume-7, Issue-6S, March 2019.

[17] U., V., Choudhari, E., Deshpande, P., \& Chaudhary, P. "A Survey To Improve Quality Of Service For Mobile Ad Hoc Networks," International Journal of Scientific Research And Education, Vol.6, Issue 8, August 2018.

[18] S. K., R. V., R. M. B., S. H., S. M., "A Comprehensive Study on Wireless Ad-Hoc Networks," International Journal of Advanced Research in Electrical, Electronics and Instrumentation Engineering, Vol. 8, Issue 1, January 2019.

[19] S. Singh, A. K. Singh, "DSR PROTOCOL FOR DATA TRANSMISSION," International Journal Of Inventive Research In Science, Volume 1, Issue 1, June 2019.

[20] P. Sharma, "Integration of Mobile IP with MANET," International Journal of Computer Trends and Technology (IJCTT), Volume 39, Number 1, September 2016.

[21] T. Issarivakul. and E. Hossain. "Introduction to network simulator 2 (NS2)," An Introduction to network simulator NS2 (pp. 1-18). Springer, Boston, MA, 2009.

[22] G., G., S., V., K., A. \& M., U.. Current Approaches on Gastro Retentive Drug Delivery System: An Overview. International Journal of Pharmacy Research \& Technology, 16-28, 2019.

[23] K. Harju, and S. Korventausta, "Network simulation and protocol implementation using network simulator 2," TTKK, 2001.

[24] M. M. Parvez, S. Chowdhury, and S. M.Bulbul, "Improved comparative analysis of Manet," $14^{\text {th }}$ May 2012.

[25] T. Issariyakul and E. Hossain "Introduction to Network Simulator NS2" ISBN: 978-0-387-71759-3.

[26] K., B.. Nitrate reductase enzyme in Escherichia coli and its relationship with the synthesis of silver nanoparticles. UCT Journal of Research in Science, Engineering and Technology, 26-32, 2015. 